\title{
Justification of the mathematical (thermodynamic) model of deformation processes in the materials cutting system
}

\author{
Natalia Neumoina ${ }^{*}$, Alexander Ivashchenko ${ }^{1}$, and Alexander Belov \\ Kamyshin Technology Institute (branch) of the Volgograd State Technical University, 403874 \\ Kamyshin, Russia
}

\begin{abstract}
By the cutting system we mean the workpiece, shavings and the cutting tools that are in the conditions of contact interaction. In this system occur: the destruction of the material; elastic and plastic deformation; the formation of a new surface; electric and magnetic polarization of the surface layers; mechanical interaction of components of the cutting system, accompanied by internal and external friction; heat exchange between the components of the cutting system, which involves all the existing methods of heat transfer in nature (convection, heat conductivity, radiation); the withdrawal of energy from the system, accompanied by the formation of so-called dissipative structures. The authors propose a mathematical model developed using the thermodynamic theory, which takes into account the mutual transformation of energy in the system. To describe highly nonequilibrium processes, it is necessary to determine not only the state of the system at one time or another, but also be able to calculate the irreversible energy flows (the flux of the released elastic energy, the work flow of destruction, the mass flow, etc.), through which it is possible to trace changes, occurring in the cutting system.
\end{abstract}

\section{Introduction}

Processing materials by cutting to date is one of the most common molding processes of machine parts having the desired dimensions and quality of newly formed surface. By the cutting system, we mean the workpiece, shavings and the cutting tools that are in the conditions of contact interaction.

In this system occur: the destruction of the material; elastic and plastic deformation; the formation of a new surface; electric and magnetic polarization of the surface layers; mechanical interaction of components of the cutting system, accompanied by internal and external friction; heat exchange between the components of the cutting system, which involves all the existing methods of heat transfer in nature (convection, heat conductivity, radiation); the withdrawal of energy from the system, accompanied by the formation of socalled dissipative structures. In addition, all these processes have a dynamic and cyclical nature. Also, during the course of these processes, the signs of self-organization are clearly

* Corresponding author: neumiona@kti.ru 
manifested (the system is thermodynamically open, deviations from equilibrium exceed critical values, there is a hierarchical complexity of phenomena, macroscopic processes proceed in concert). The complexity of the joint description of these physical processes in the machining zone is obvious.

Let's consider in more detail one of the above-mentioned features the process cutting of materials, namely the dynamic nature of the process and the occurrence of vibrations in the cutting system. In works [1,2] the analysis of the reasons of occurrence of vibrations at cutting of materials. One of the significant reasons for the authors of $[1,2]$ is the delay in the cutting forces with respect to perturbations during the deformation of the metal in the cutting zone. The introduction of the cutting wedge of the tool is accompanied by compression of the processed material (Fig. 1).

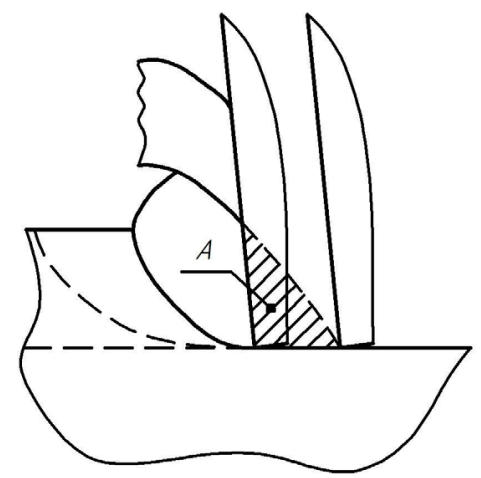

Fig.1. The process of formation shavings.

When the critical value of the effective stress is reached at the cutting edge, the cut off layer is separated from the workpiece material. At this moment, the second stage of the elementary act of shavings formation begins - the shear stage. The formed element (element A) of shavings moves along the shear surface with high acceleration and an intense decrease in shear resistance, and also up along the front surface. In this case, it undergoes additional deformations that lead to a non-uniform hardening of the shavings along the section and create the prerequisites for its twisting. Then the shift stops, and the movement of the shavings element along the front surface becomes more uniform and continues until it is removed from the contact zone.

Due to the difference in the nature of the deformation processes under the elementary act of shavings formation, the cutting force is not constant and undergoes periodic changes (Fig. 2).

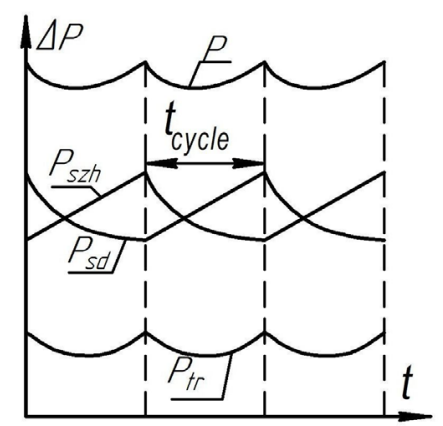

Fig.2. Change in cutting force in the act of the shavings formation. 
At the moment, when the shavings element starts to move, the next element begins, i.e. the new local area of the processed material is compressed. Thus, the first and second stages of the process begin and end simultaneously, but relate to two neighboring shavings elements. The presented qualitative picture of the elementary act of formation of the shavings of a cyclic character takes place practically independently at cutting. The main parameters of cutting (speed, cutting depth and feed) and the properties of the material being processed also have a significant effect on the nature of deformation processes. As a result, the volume of the deformation zone, the compression rate, the shear rate, the rate of hardening and weakening of the cutting system elements vary. A very significant influence on these processes is due to the rate of heat exchange and mass exchange between the elements of the cutting system. This leads to the formation of different types of shavings and different qualities of cutting surface.

\section{Justification of the model}

One can write the energy balance equation for the cutting system in the form:

Where

$$
E_{M}=E_{P}+E_{D}
$$

$$
E_{D}=E_{1}+E_{2}+E_{3}+E_{4}+E_{5}+E_{6}
$$

In equation (1), $E_{M}$ is the mechanical power of the cutting process, $E_{P}$ is the useful energy expended for cutting, and $E_{D}$ is the energy dissipated. Scattering of mechanical energy in the cutting system can occur in various ways, (equation (2)), this is the strain energy that is stored in the chips, cutting surfaces of the tool and the surface layer of the processed billet $\left(E_{l}\right)$; elastic energy released as an acoustic emission signal $\left(E_{2}\right)$; thermal energy released due to plastic deformation and friction of the elements of the cutting system $\left(E_{3}\right)$; energy of motion of dislocations $\left(E_{4}\right)$; energy, going to mass transfer $\left(E_{5}\right)$ and chemical transformations $\left(E_{6}\right)$ in near surface layers of billet, shavings and tools.

One of the reasons why the process of cutting materials can be attributed to selforganizing is that the energy flows on the right side of equation (2) lead to the formation of new spatio-temporal structures called dissipative structures. A significant deviation of the system from the state of equilibrium is a necessary, but insufficient condition for the formation of such structures. An important factor is the coherent behavior of the elements of the system. Thus, by dissipative structures, we mean at present such neoplasms in the system that can arise as a result of the presence of a qualitative jump when critical (threshold) values of the system parameters are reached. The dissipative structures in the cutting system include: fragmented dislocation structure, slip bands localized in the space of the plastic flow zone, amorphous crystal structures in the surface layers of the chips and billets, twisted chips, build-up, crack formation zone near of the cutting edge.

It follows from the foregoing that each of the energy flows of the right-hand side of equation (2) is irreversible. The flow of heat and the flow of mass resist the flow of destruction. The development of the process of plastic deformation and its result is determined by the ratio of the energy fluxes of metal destruction, as well as energy flows opposing this destruction, that is, which of these flows will prevail. It was shown in [3] that the greater the entropy flux density and the shorter the relaxation time, the greater the possibility of realizing this irreversible process in comparison with others. At the same entropy density, the process with the smallest relaxation time is most probable. The authors of [4] also come to a similar conclusion. And they consider a strongly deformed metal as a developed hierarchical system. In such a system, there is a spectrum of relaxation times and first, faster processes take place that are responsible for overcoming potential barriers of 
minimum height. The hierarchical nature of the processes lies in the fact that until the channels with the minimal relaxation time are realized, the network of channels of the next level is not included. In Fig. 3 [4] is a diagram of the form of the potential energy of a three-level hierarchical system. As an example of such behavior, one can also cite the experimentally noted fact, which consists in the simultaneous formation of structures $(i+1)$ and (i-1) ranks during plastic deformation of samples made of epoxy resin, i.e. simultaneously compound and crushing of blocks [5].

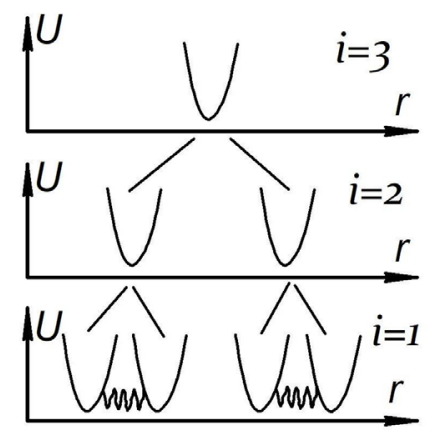

Fig. 3. Type of potential energy of the three-level hierarchical system.

In connection with the foregoing, it is of particular interest to estimate the relaxation times of the energy flows that accompany the process of destruction and plastic deformation of the metal [3]. Data for calculation are taken from reference books [6,7]. The process of releasing elastic energy is characterized by time (Fig. 4):

$$
\tau_{y}=l \sqrt{ }\left(2 \rho E / \sigma^{2}\right)
$$

Where: $l$ - the critical size of the crack; $\rho$ - the density of the metal; $E$ - the modulus of elasticity of the first kind; $\sigma$ - stress that occurs in the material during the development of a crack.

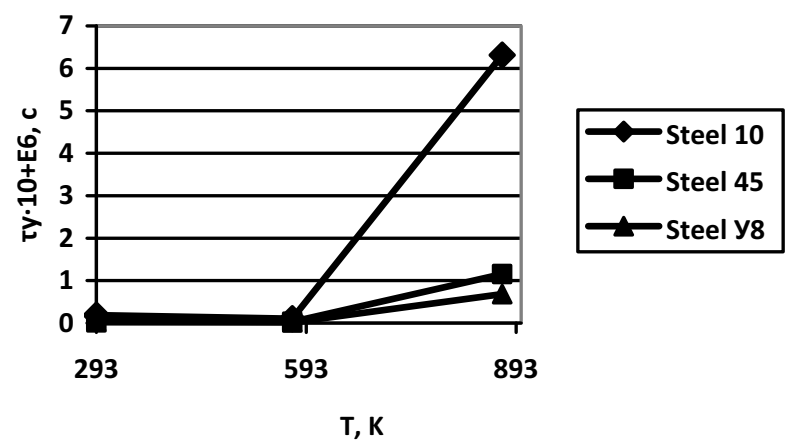

Fig. 4. The relaxation time of elastic energy. theory:

The critical size of the crack can be calculated from the formula given by the Griffith

$$
l=2 E \gamma /\left(\pi \sigma^{2}\right)
$$

Where: $\gamma$ - specific energy of surface failure.

The process of crack formation is characterized by time (Fig. 5): 


$$
\tau_{l}=l \sqrt{ }(\rho l / \gamma)
$$
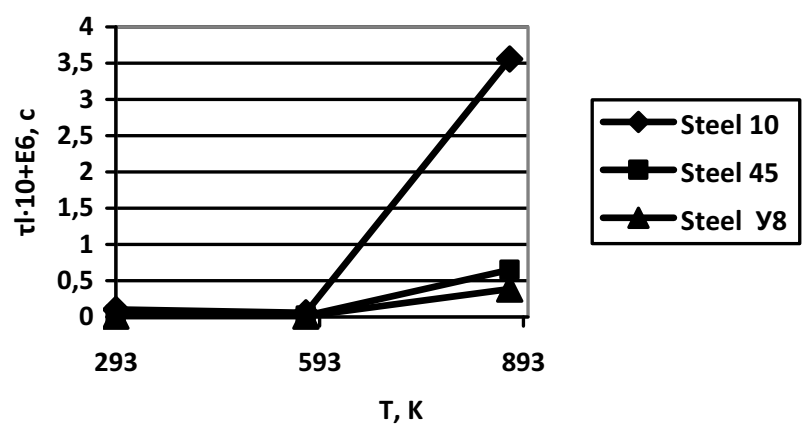

Fig. 5. Time of crack formation.

The process of heat removal is characterized by time (Fig. 6):

$$
\tau_{\lambda}=l^{2} / a
$$

Where: $a$ - coefficient of thermal diffusivity.
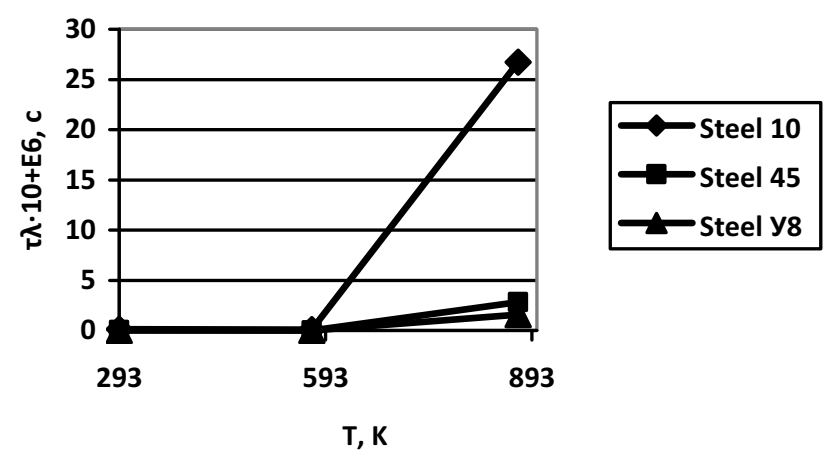

Fig. 6. Heat removal time.

Knowing the relaxation time of the processes that develop during the plastic deformation of a metal, it is possible to reveal the characteristic features of metal deformation under given conditions, up to the establishment of quantitative bonds.

Within the framework of the thermodynamic theory, one can take into account the features of the mechanical and thermal interaction of the elements, the hierarchical nature of the deformation processes, taking into account the mutual transformation of energy in the cutting system. As such a theory, it is proposed to use the nonlocal version of thermodynamics developed by V. Maikov [8].

Nonlocal thermodynamics, being in its nature deductive, is based on the assertion that there exists in nature a quantum of entropy equal to the Boltzmann constant. If we take the value of $k T$ for a macroscopic definition of entropy as the minimum increment (the quantization interval) of the amount of heat:

$$
\Delta Q=k T
$$

From the definition of entropy in accordance with the second law of thermodynamics, we obtain the minimum increment of entropy: 


$$
\Delta S=k T / T=k
$$

The procedure of macro quantification leads to important consequences. The decisive role here belongs to obtaining a characteristic discrete time:

$$
\Delta t=h /(2 k T)
$$

Where: $h$ - Planck's constant.

In the new theory, the existence of a micro- and macro level boundary is shown, that is, a minimal macroscopic volume (hereinafter "macro cell") is formulated, to which the thermodynamic method is applicable. It is established that the radius and volume of a macro cell:

$$
\begin{gathered}
r=c \Delta t=c h /(2 k T) \\
V=(4 / 3) \pi r^{3}=(\pi / 6)(c h / 2 k T)^{3}
\end{gathered}
$$

Where: $c$ - light speed in vacuum.

The radius $r$ and volume $V$ of the macro cell determine the dimensions of the space in which the local thermodynamic equilibrium is established in the dynamically equilibrium system, hence, the temperature is formed as a macroscopic parameter.

For example, at $T=300 \mathrm{~K}, r=3.8+E 10^{-6} \mathrm{~m}, \Delta t=1.27+E 10^{-14} \mathrm{~s}$. A macro cell can be considered as a short-lived (flickering) physical cluster, that is, a supramolecular level in the hierarchy of a macroscopic system. In nonlocal thermodynamics it is proved that the procedure of macro quantification translates the description from the region of classical static equilibrium (thermostatic) to the dynamic equilibrium region with the fluctuation interaction of the macro cell with the environment. Parameters of a macro cell (temperature, pressure, etc.) under dynamic equilibrium for a characteristic time $\Delta t$ differ from the parameters of its environment, and in this sense any material medium is inhomogeneous in thermodynamics. This kind of inhomogeneity leads to the appearance on the boundary of a macro cell with an environment of fluctuating stresses that are similar in nature to surface phenomena. Using the relations of the classical thermodynamics of deformations shows that, due to the discreteness of the spatial and time intervals, the macro cell is subjected to volume deformation and shear deformation, separated in space and time on the scale of a macro cell. In turn, the volume deformation causes electrical polarization, and the shear magnetic. Polarization leads to the appearance of bound charges of electric and magnetic types. These phenomena form a thermic deformation equilibrium cycle of a macro cell (Fig 7).

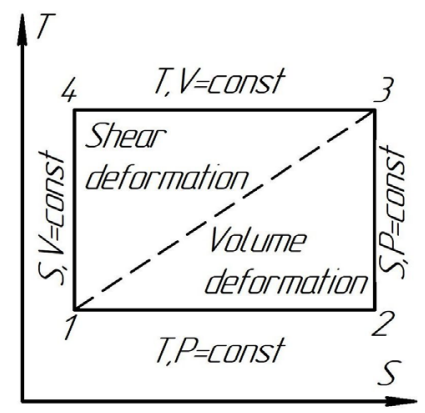

Fig. 7. Thermic deformation equilibrium cycle of a macro cell.

Let us illustrate by an example the possibility of applying the above theory to the description of deformation processes in the cutting zone. 
We calculate the normal and tangential stresses in the machining zone and compare them with the experimental values of stresses obtained on the chamfer of the back surface of the tool. In the experiment [9] at the maximum value of the force $P_{y}=3200 \mathrm{~N}$, and the force $P_{z}=1600 \mathrm{~N}$, the corresponding stresses are $\sigma=1600 \mathrm{MPa}, \tau=400 \mathrm{MPa}$.

Calculated Values:

$$
\begin{aligned}
\sigma & =P_{y} \Delta x / V=3200 \cdot 6.57 E^{-11} / 2.33 E^{-16}=901.7 \mathrm{MPa} \\
\tau & =P_{z} l / \Delta V^{\prime}=1600 \cdot 6.04 E^{-19} / 2.36 E^{-24}=409.5 \mathrm{MPa}
\end{aligned}
$$

As the calculations show, using concepts specific for the nonlocal version of thermodynamics, such as characteristic linear dimensions for the volume deformation $\Delta x$ and shear deformation $l$, as well as the volume of the macro cell $V$ and the elementary change in the volume of the macro cell in result of the deformation $\Delta V^{\prime}$, one can get an order of magnitude of the stresses, observed in the experiment.

In the framework of the nonlocal version of thermodynamics, expressions for calculating the mass and heat flux have already been obtained, which have been tested experimentally in the modeling of the heat and mass transfer process [10,11].

In the framework of the nonlocal version of thermodynamics one can also obtain equations for the flow of elastic energy at nonequilibrium volume deformation:

$$
J_{V d}=u \cdot \sigma \cdot c_{S}
$$

and the flow of elastic energy for nonequilibrium shear deformation:

$$
J_{S d}=\gamma \cdot \tau \cdot c_{S}
$$

Where: $c_{S}$ - speed of sound in a given medium, $u$ and $\gamma$ - volumetric and shear deformation parameters.

\section{Conclusion}

Thus, based on the above, we can draw the following conclusions:

- The non-local version of thermodynamics reasonably determines the minimum macroscopic volume characterizing the collective behavior of the medium;

- The definition of this volume allows us to proceed consistently to a hierarchical examination of deformation processes in a solid;

- Within the framework of the above theory, the cycling of natural processes (the thermo-deformation cycle of a macro cell) is taken into account;

- Within the framework of the thermal deformation cycle, it is possible to relate mechanical, thermal and electrodynamic phenomena, which is actually observed in nature.

All of the above will allow us to proceed to a description of that complex of processes and phenomena that accompany the process of cutting materials.

Further, to create an adequate mathematical thermodynamic model, it is necessary to solve a number of problems. First, to determine the patterns of high-speed deformation of metals with the definition of the scale of the deformation zone, depending on the parameters of cutting. Secondly, to determine the patterns of the process from the point of view of the hierarchy of structures under destruction, i.e. for a specific set of cutting parameters, determine the "mechanism" of the process. Thirdly, to learn to determine the relaxation times for various energy flows and with the help of this information to model the behavior of elementary volumes during the destruction and deformation of the material.

Thus, it can be concluded that today there are prerequisites for developing a thermodynamic approach to describing deformation processes at the cutting materials. 


\section{References}

1. V. Veits, V. Maxarov, P. Lontsikh, Dynamics and modelling of cutting processes during machining (Irkutsk, 2000)

2. L. Filimonov, L. Petrashina, Bulletin of Machine Building. 5-6, 23 (1993)

3. I. Novikov, Sat. Physico-mechanical and thermophysical properties of metals, 170 (1976)

4. R. Il'kaev, A. Uchaev, S. Novikov, DAN, 3, 328 (2002)

5. R. Goldstein, V. Osipenko, DAN, 4, 735 (1992)

6. M. Shishkov, Marochnik of steels and alloys: Reference book (Yugo-Vostok, 2002)

7. A.V. Bobylev, Mechanical and technological properties of metals: Handbook (Metallurgy, Moscow, 1980)

8. V. Maikov, The extended version of classical thermodynamics is the physics of discrete space-time (MGUIE, Moscow, 1997)

9. M. Poletika, V. Kozlov, The collection of works, Progressive technological processes in mechanical engineering. Tomsk, 18 (1997)

10. N.G. Neumoina, A.V. Belov, J. Modern problems of science and education, 6, 26 (2014)

11. N.G. Neumoina, A.V. Belov, J. Modern problems of science and education, 6, 27 (2014) 